\title{
Interstellar Dust Inside and Outside the Heliosphere
}

\author{
Harald Krüger · Eberhard Grün
}

Received: 5 February 2008 / Accepted: 5 August 2008 / Published online: 26 September 2008

(C) Springer Science+Business Media B.V. 2008

\begin{abstract}
In the early 1990s, after its Jupiter flyby, the Ulysses spacecraft identified interstellar dust in the solar system. Since then the in-situ dust detector on board Ulysses continuously monitored interstellar grains with masses up to $10^{-13} \mathrm{~kg}$, penetrating deep into the solar system. While Ulysses measured the interstellar dust stream at high ecliptic latitudes between 3 and $5 \mathrm{AU}$, interstellar impactors were also measured with the in-situ dust detectors on board Cassini, Galileo and Helios, covering a heliocentric distance range between 0.3 and $3 \mathrm{AU}$ in the ecliptic plane. The interstellar dust stream in the inner solar system is altered by the solar radiation pressure force, gravitational focussing and interaction of charged grains with the time varying interplanetary magnetic field. The grains act as tracers of the physical conditions in the local interstellar cloud (LIC). Our in-situ measurements imply the existence of a population of 'big' interstellar grains (up to $10^{-13} \mathrm{~kg}$ ) and a gas-to-dust-mass ratio in the LIC which is a factor of 1.5-2 larger than the one derived from astronomical observations, indicating a concentration of interstellar dust in the very local interstellar medium. Until 2004, the interstellar dust flow direction measured by Ulysses was close to the mean apex of the Sun's motion through the LIC, while in 2005, the data showed a $30^{\circ}$ shift, the reason of which is presently unknown. We review the results from spacecraft-based in-situ interstellar dust measurements in the solar system and their implications for the physical and chemical state of the LIC.
\end{abstract}

Keywords Dust · Interstellar dust · Heliosphere · Interstellar matter

\footnotetext{
H. Krüger $(\bowtie)$

Max-Planck-Institut für Sonnensystemforschung, Max-Planck-Str. 2, 37191 Katlenburg-Lindau, Germany

e-mail: krueger@linmpi.mpg.de

H. Krüger · E. Grün

Max-Planck-Institut für Kernphysik, Saupfercheckweg 1, 69117 Heidelberg, Germany

E. Grün

Laboratory for Atmospheric and Space Physics, University of Colorado, Boulder, CO, 80303-7814, USA
} 


\section{Introduction}

Interstellar dust (ISD) became a topic of astrophysical research in the early 1930s when the existence of extinction, weakening, and scattering of starlight in the interstellar medium (ISM) was realised. At that time, astronomical observations provided the only information about the properties of the dust in the ISM. With the advent of dust detectors onboard spacecraft, it became possible to investigate dust particles in-situ. About 30 years ago, analysis of the data obtained with the dust instruments flown on a couple of spacecraft suggested that ISD grains can cross the heliospheric boundary and penetrate deeply into the heliosphere (Bertaux and Blamont 1976; Wolf et al. 1976). In the 1990s, this was undoubtedly demonstrated with the dust instrument carried by the Ulysses spacecraft: the Ulysses dust detector, which measured mass, speed and approach direction of the impacting grains, identified ISD grains with radius above $0.1 \mu \mathrm{m}$ sweeping through the heliosphere (Grün et al. 1993, 1994, 1995).

The galactic setting of the Sun is dominated by the Local Bubble void in the interstellar medium which is a low-density, presumably hot region of irregular shape that extends to approximately $100 \mathrm{pc}$ from the Sun. The Local Bubble was excavated by supernova explosions and winds of massive stars in the neighbouring starforming regions of the ScorpiusCentaurus and Orion Associations. The hot gas in the Local Bubble is usually called Local Interstellar Medium (LISM). Figure 1 shows the projection in galactic coordinates of 15 interstellar clouds identified within $15 \mathrm{pc}$ of the Sun. According to recent analyses (Redfield and Linsky 2008) the Sun appears to be located in the transition zone between two of the closest clouds, the Local Interstellar Cloud (LIC) and the G cloud. The only direct observation of ISD close to the Sun is weak polarization observed along the sightline towards $36 \mathrm{Oph}$ (distance about $6 \mathrm{pc}$ ) which is due to magnetically aligned dust grains (Tinbergen 1982). Therefore, in-situ sampling of dust from the LIC can greatly improve our understand-

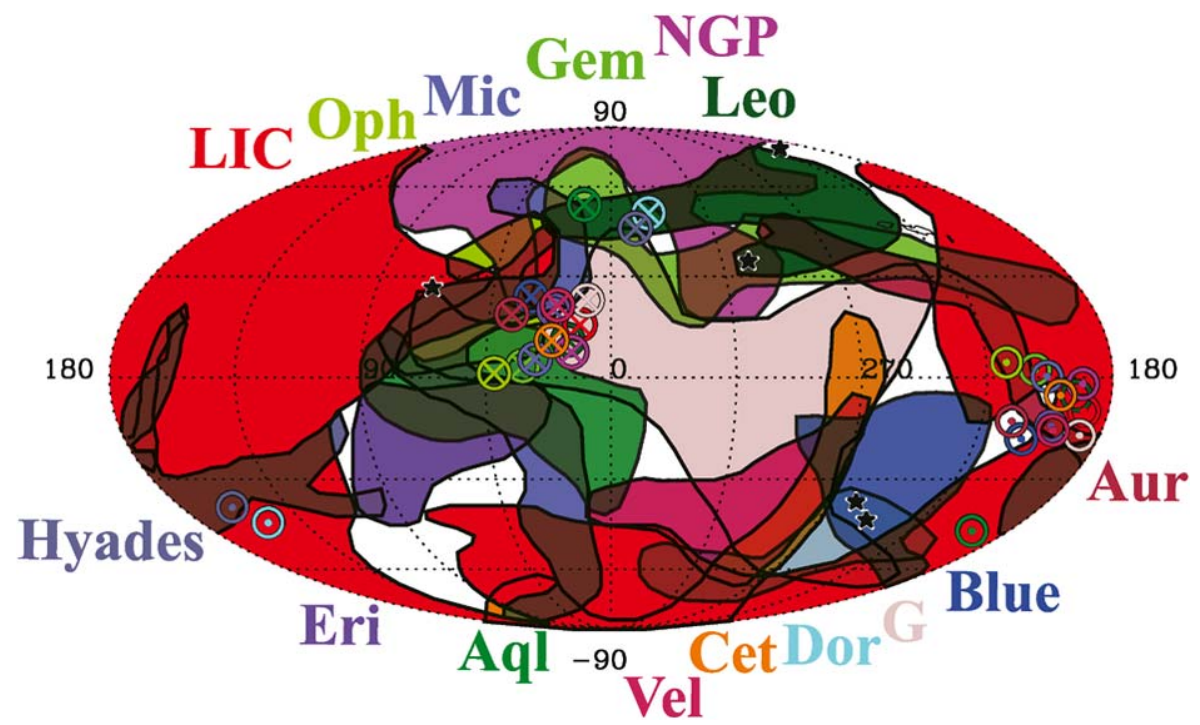

Fig. 1 Locations in galactic coordinates of 15 interstellar clouds located within 15 pc of the Sun (from Redfield and Linsky 2008). The upwind heliocentric direction of the velocity vector for each cloud is indicated by the circled cross, while the downwind heliocentric direction is indicated by a circled dot 
ing of the nature and processing of dust in various galactic environments and can cast new light on the chemical composition and homogeneity of the interstellar medium.

In this paper we review the results from in-situ ISD measurements obtained with the Ulysses and other space-borne dust detectors. We review our current knowledge about ISD inside the heliosphere and in our local interstellar environment.

\section{Interstellar Dust Inside the Heliosphere}

The Ulysses in-situ dust measurements showed that the grain motion through the solar system is parallel to the flow of neutral interstellar hydrogen and helium gas, both gas and dust travelling with a speed of $26 \mathrm{~km} \mathrm{~s}^{-1}$ (Grün et al. 1994; Baguhl et al. 1995; Witte et al. 1996; Witte 2004). The upstream direction of the dust flow lies at $259^{\circ}$ ecliptic longitude and $8^{\circ}$ latitude (Landgraf 1998). The interstellar dust flow persists at high ecliptic latitudes above and below the ecliptic plane and even over the poles of the Sun, whereas interplanetary dust is strongly depleted at high latitudes (Grün et al. 1997). The interstellar dust flux measured at a distance of about $3 \mathrm{AU}$ from the Sun is time-dependent, and the mean mass of the grains is about $3 \times 10^{-16} \mathrm{~kg}$ (Landgraf et al. 2000), corresponding to a grain radius of approximately $0.3 \mu \mathrm{m}$. Measurements with the identical dust instrument onboard Galileo performed in the ecliptic plane showed that beyond about $3 \mathrm{AU}$ the interstellar dust flux even exceeds the flux of micron-sized interplanetary grains.

Results from the first decade of the Ulysses mission showed that the radii of clearly identified interstellar grains range from $0.05 \mu \mathrm{m}$ to above $1 \mu \mathrm{m}$. The data show distancedependent alteration of the interstellar dust stream caused by gravitational focussing by the Sun, solar radiation pressure, and electromagnetic interaction with the time-varying interplanetary magnetic field (IMF; Altobelli et al. 2003, 2005a, 2005b; Mann and Kimura 2000; Landgraf 2000; Czechowski and Mann 2003). Radiation pressure and electromagnetic forces strongly depend on grain size, leading to a strong modification of the size distribution and fluxes of grains measured inside the heliosphere (Landgraf et al. 1999, 2003). Strong filtration of small grains due to electromagnetic forces also occurs at the heliospheric boundary (Linde and Gombosi 2000). The overall size distribution measured in-situ with Ulysses within 5 AU from the Sun shows a deficiency of small grains below $0.3 \mu \mathrm{m}$ compared to astronomically observed ISD (Mathis 2000; Draine 2003; Frisch et al. 1999). In addition, solar radiation pressure deflects grains with sizes of about $0.4 \mu \mathrm{m}$ and was found to be effective at solar distances below 4 AU (Landgraf et al. 1999).

Significant differences in the particle sizes were also recorded at different heliocentric distances. In addition to the Ulysses measurements which revealed a lack of small $0.3 \mu \mathrm{m}$ ISD grains within $3 \mathrm{AU}$ heliocentric distance, measurements by Cassini and Galileo between 0.7 and $3 \mathrm{AU}$ showed that the detected interstellar particles were bigger than $0.5 \mu \mathrm{m}$, with grain masses increasing closer to the Sun (Altobelli et al. 2003, 2005b; Altobelli 2004). The flux of these bigger particles did not exhibit temporal variations due to the solar-wind magnetic field like the flux of smaller particles observed by Ulysses. The trend of increasing particle masses continues even closer to the Sun, as demonstrated by Helios which recorded particles of about $1 \mu \mathrm{m}$ down to $0.3 \mathrm{AU}$ (Altobelli et al. 2005a, 2006). These facts support the idea that the ISD stream is strongly filtered by solar radiation pressure. Interstellar particles with optical properties of astronomical silicates or organic refractory materials are consistent with the observed radiation pressure effects (Landgraf et al. 1999).

In addition to studies of the distribution of grain masses, the Ulysses dust instrument monitored the flux of the interstellar particles in the heliosphere (Fig. 2). In mid 1996, we 
Fig. 2 Fit of simulated to measured flux (Landgraf et al. 2003). The fit parameters are the relative contributions of grains of sizes between 0.1 and $0.4 \mu \mathrm{m}$ (the $0.1 \mu \mathrm{m}$ curve is not shown, because it did not contribute to the fit). The solid lines show the flux profiles of the simulated grains of various sizes, scaled with their best-fit relative contributions. The shaded region indicates the best-fit total predicted flux, with its vertical extent giving the $1 \sigma$ uncertainty

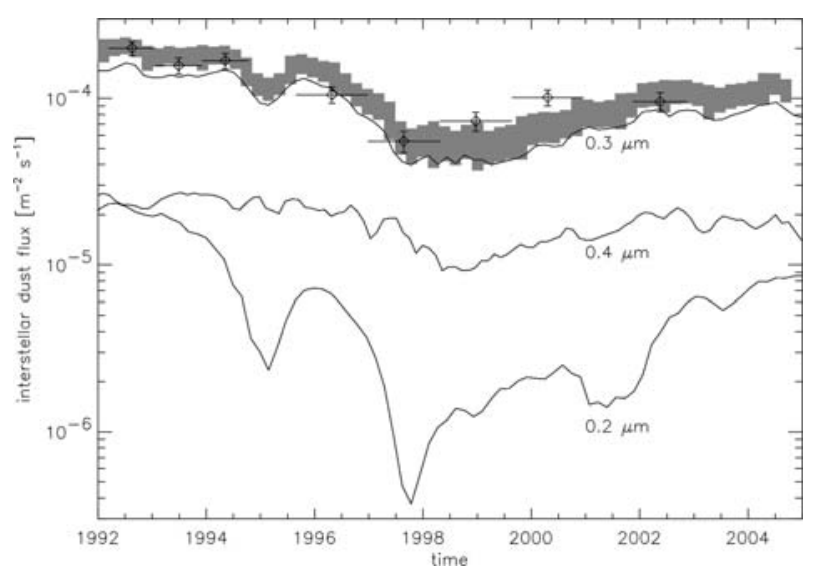

observed a decrease of the interstellar dust flux by a factor of 3 from an initial value of $1.5 \times$ $10^{-4} \mathrm{~m}^{-2} \mathrm{~s}^{-1}$ down to $0.5 \times 10^{-4} \mathrm{~m}^{-2} \mathrm{~s}^{-1}$. This drop was attributed to increased filtering of small grains by the solar wind driven IMF during solar minimum conditions (Landgraf 1998, 2000; Landgraf et al. 2000). Since early 2000, Ulysses has again detected interstellar dust flux levels above $10^{-4} \mathrm{~m}^{-2} \mathrm{~s}^{-1}$ (Landgraf et al. 2003; Krüger et al. 2007). Monte-Carlo simulations of the grain dynamics in the heliosphere showed that the dominant contribution to the dust flux comes from grains with a charge-to-mass ratio of $q / m=0.59 \mathrm{C} \mathrm{kg}^{-1}$ and a radiation pressure efficiency of $\beta=1.1$, corresponding to grain radii of $0.3 \mu \mathrm{m}$ (Landgraf et al. 2003).

Particles even bigger $(40 \mu \mathrm{m})$ than the grains measured in-situ with the spacecraft detectors were reliably identified by meteor radar observations (Taylor et al. 1996; Baggaley 2000; Baggaley and Neslušan 2002; Meisel et al. 2002). The grains were identified by their hyperbolic speeds, and their flow direction varied over a significantly wider angular range than that of the much smaller grains observed by spacecraft. Baggaley (2000) identified a general background influx of extra-solar system particles from southern ecliptic latitudes with enhanced fluxes from discrete sources. More sensitive meteor observations with the Arecibo radar found micron-sized interstellar meteor particles radiating from the direction of the Geminga pulsar (Meisel et al. 2002). This is particularly interesting because the supernova that formed the Geminga pulsar is a potential candidate which may have created the Local Bubble.

Ulysses has monitored the interstellar dust flow through the solar system for more than 15 years now. This time period covers more than two and a half revolutions of the spacecraft about the Sun through more than $2 / 3$ of a complete 22-year solar cycle. Thus, Ulysses measured interstellar dust during solar minimum and solar maximum conditions of the interplanetary magnetic field (IMF). The interstellar dust flux modulation due to grain interaction with the magnetic field during solar minimum could be well explained (Landgraf 1998, 2000; Landgraf et al. 2003). By taking into account the sensor side wall in the instrument field of view we could recently improve the flux determination (Altobelli et al. 2004).

Until early 2005 the approach direction of the interstellar grains was in agreement with the interstellar helium flow direction as determined by the Ulysses/GAS measurements (Witte 2004; Witte et al. 2004; Landgraf and Grün 1998; Frisch et al. 1999; Krüger et al. 2006). An example is shown in the left panel of Fig. 3 which shows the impact direction of the interstellar impactors in the period from 1996 to 2000. Six years 

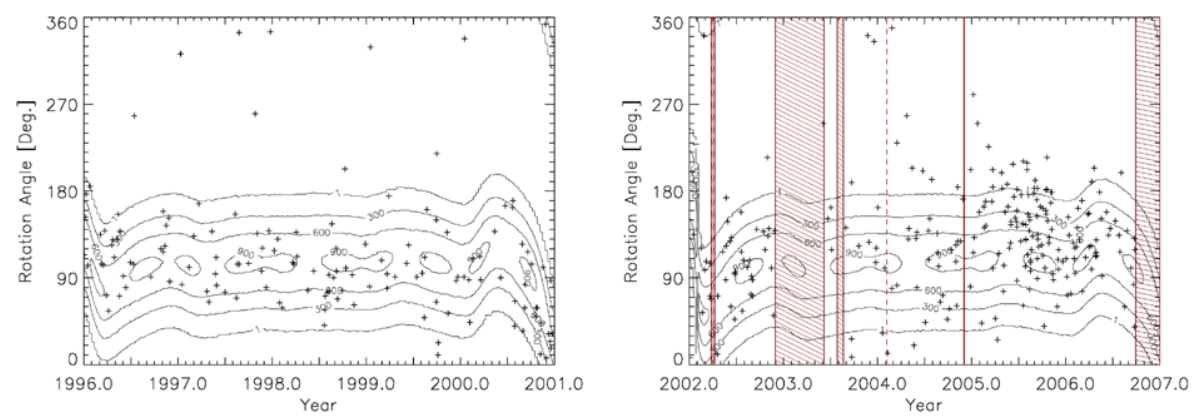

Fig. 3 Impact direction (i.e. spacecraft rotation angle at dust particle impact) of interstellar grains measured with Ulysses in two time intervals (from Krüger et al. 2007). Each cross indicates an individual dust impact. Left: 1 January 1996 to 31 December 2000; right: 1 January 2002 to 31 December 2006. In the time intervals shown here, the spacecraft rotation axis was pointing towards Earth. The dust detector was mounted almost perpendicular to the spacecraft-Earth line so that it scanned through a plane perpendicular to the spacecraft-Earth line (Grün et al. 1992, 1997; Krüger et al. 2001, 2006). Ecliptic north is close to $0^{\circ}$. Contour lines show the effective sensor area for particles approaching from the upstream direction of interstellar helium (Witte 2004; Witte et al. 2004). In the right panel, a vertical dashed line shows Jupiter closest approach on 5 February 2004, five shaded areas indicate periods when the dust instrument was switched off

later, when Ulysses was travelling through almost the same spatial region and had an almost identical detection geometry for interstellar grains, the situation was vastly different: first, the range in approach directions of the grains was somewhat wider (best seen in 2004; right panel of Fig. 3; see also Krüger et al. 2007); second, and more noticeable, in 2005/06 the approach direction of the majority of grains was shifted away from the helium flow direction. Our preliminary analysis indicates that this shift is about $30^{\circ}$ away from the ecliptic plane towards southern ecliptic latitudes (Krüger et al. 2007). At the moment, we do not know whether it is a temporary shift limited to the time period stated above or whether it continues to the present time. Furthermore, the reason for this shift remains mysterious. Whether it is connected to a secondary stream of interstellar neutral atoms shifted from the main neutral gas flow (Collier et al. 2004; Wurz et al. 2004; Nakagawa et al. 2006) is presently unclear. Given, however, that the neutral gas stream is shifted along the ecliptic plane while the shift in the dust flow is offset from the ecliptic, a connection between both phenomena seems unlikely.

Even though Ulysses' position in the heliosphere and the dust detection conditions were very similar during both time intervals considered in Fig. 3, the configurations of the solar wind driven interplanetary magnetic field (IMF), which strongly affects the dynamics of the smallest grains, were completely different. We have to consider that the interstellar grains need approximately twenty years to travel from the heliospheric boundary to the inner solar system where they are detected by Ulysses. Thus, the effect of the IMF on the grain dynamics is the accumulated effect caused by the interaction with the IMF over several years: In the earlier time interval (1997-1999) the grains had a recent dynamic history dominated by solar minimum conditions (Landgraf 2000), while the grains detected during the second interval (2002-2005) had a recent history dominated by the much more disturbed solar maximum conditions of the IMF. During the solar maximum conditions the overall magnetic dipole field changed polarity. Morfill and Grün (1979) predicted that due to this effect in a 22-year cycle, small interstellar grains experience either focussing or defocusing conditions. During these times they are systematically deflected by the solar wind magnetic field either towards or away from the solar magnetic equator plane (close to the ecliptic plane). This latter configuration likely has a strong influence on the dust dynamics and the total interstellar flux 
in the inner heliosphere but it is not modelled in detail in the presently existing models. An explanation of the grain interaction with the IMF at the recent solar maximum conditions is still pending.

The fact that the models fit the flux variation by assuming a constant dust concentration in the Local Interstellar Cloud (LIC) implies that the dust phase of the LIC must be homogeneously distributed over length scales of $50 \mathrm{AU}$, which is the distance inside the LIC travelled by the Sun during the measurement period of Ulysses from end 1992 to end 2002 (Landgraf et al. 2003). This conclusion is supported by the more recent Ulysses data until the end of 2004 (Krüger et al. 2006). The 2005/06 data, on the other hand, put a question mark onto this conclusion because if the observed shift in impact direction turns out to be intrinsic, it would imply that this homogeneity breaks down on larger length scales.

\section{Interstellar Dust in the Local Interstellar Cloud}

ISD grains carry information about their past dynamics outside the heliosphere and are thus of strong interest to understand the dynamical processes in the Local Interstellar Cloud (LIC). They provide the main reservoir and transport mechanism of heavy elements in the interstellar medium ( $\mathrm{Li}$ and Greenberg 1997). The dynamics of the grains is crucial for understanding of nucleation, growth and collisional destruction processes (Draine 2003) which strongly depend on the relative velocities of the grains. The most important phenomena responsible for the spread of velocities in the LIC are gas drag, interaction with the local interstellar magnetic field, radiation pressure and photoelectric emission (Frisch et al. 1999). The relative strengths of the different forces strongly depend on the size and the charge of the grains, together with local conditions of the interstellar medium (ISM), like gas or magnetic field turbulences.

Observations of interstellar material (ISM) towards nearby stars and inside of the solar system, combined with radiative transfer models, constrain the conditions in the LIC (Frisch 1998, 1999; Frisch and Slavin 2003; Slavin and Frisch 2006, 2007, 2008). To our present knowledge, the main characteristics of the LIC are: atomic neutral hydrogen density $\mathrm{n}\left(\mathrm{H}^{0}\right)=0.19-0.20 \mathrm{~cm}^{-3}$, electron and ion density $\mathrm{n}\left(\mathrm{e}^{-}\right)=0.05-0.08 \mathrm{~cm}^{-3}$, temperature $\sim 6300 \mathrm{~K}$, and a relative Sun-cloud velocity $\sim 26 \mathrm{~km} \mathrm{~s}^{-1}$. The physical conditions in the LIC are those of the intercloud medium — warm, low density, partially ionized gas $\left(X\left(\mathrm{H}^{+}\right) \sim 0.2\right.$, $X\left(\mathrm{He}^{+}\right) \sim 0.4$ ). An enhancement of refractory elements (such as $\mathrm{Fe}, \mathrm{Mg}, \mathrm{Mn}$ ) in LIC gas, compared to cool interstellar clouds, points to the destruction of interstellar dust grains by interstellar shocks (velocity 100-200 $\mathrm{km} \mathrm{s}^{-1}$; Frisch et al. 1999).

At least 5 distinct cloudlets are found within $5 \mathrm{pc}$ of the Sun, with differing compositions and physical properties (Redfield and Linsky 2002, 2004a, 2004b, 2008). Temperatures range from $5,400 \mathrm{~K}$ (towards $\alpha$ Cen) to $10,000 \mathrm{~K}$ (Blue Cloud towards $\varepsilon \mathrm{CMa}$ ) and total densities from $>0.04 \mathrm{~cm}^{-3}$ (Blue Cloud towards $\varepsilon \mathrm{CMa}$ ) to possibly $>5 \mathrm{~cm}^{-3}$ (Gcloud towards $\alpha$ Cen, Frisch 2003; Gry and Jenkins 2001). The gas-phase abundance of Fe, with respect to undepleted S, varies by $\sim 50 \%$ within $3 \mathrm{pc}$ of the Sun, evidently due to grain destruction processes (Frisch and Slavin 2003).

If the ISM is chemically homogeneous, elements absent from the gas phase must be depleted onto dust grains. This argument can be used to evaluate the gas-to-dust mass ratio $R_{\mathrm{g} / \mathrm{d}}$ over the integrated LIC column, and $R_{\mathrm{g} / \mathrm{d}}$ can be compared with that of other nearby interstellar clouds. However, the required knowledge of the total chemical composition of the ISM is an elusive quantity that has not been reliably determined. A 40$50 \%$ variation in $\mathrm{Fe}^{+} / \mathrm{S}^{+}$and $\mathrm{Si}^{+} / \mathrm{S}^{+}$for the two clouds towards $\varepsilon$ CMa indicates different 
grain histories for two similar clouds within $3 \mathrm{pc}$ of each other. If atoms not observed in the gas are concentrated in the dust, $R_{\mathrm{g} / \mathrm{d}}$ can be calculated from observations of interstellar absorption lines towards nearby stars. When evidence for $60-70 \%$ subsolar abundances is included, $R_{\mathrm{g} / \mathrm{d}} \sim 600$ integrated over the diameter of the LIC (Frisch et al. 1999; Frisch and Slavin 2003). Gas-to-dust mass ratios calculated from more recent models with improved solar abundances are in the range $R_{\mathrm{g} / \mathrm{d}} \sim 149-217$, again depending on solar abundances (Slavin and Frisch 2008). Interestingly, $R_{\mathrm{g} / \mathrm{d}}$ determined from comparisons of the Ulysses in-situ measurements inside of the solar system, compared to gas densities from these models, yield $R_{\mathrm{g} / \mathrm{d}}=116-127$ (Landgraf et al. 2000; Altobelli et al. 2004). It should be emphasised that the $R_{\mathrm{g} / \mathrm{d}}$ obtained from the in-situ measurements is an upper limit, since the smallest interstellar dust grains (radii $0.1 \mu \mathrm{m}$ ) are prevented from entering the heliosphere.

The analysis of the interstellar dust mass distribution by Landgraf (1998) was based on only a few years of dust measurements from the early Ulysses mission. We are presently re-evaluating the mass distribution with the data set until 2007. Our preliminary analysis confirms the earlier results. In particular, the existence and abundance of big interstellar grains as discussed above are confirmed by the more recent data. Furthermore, after 2002 the overall dust flux has levelled off to the values before 1996 (Krüger et al. 2007).

Overall, the in-situ value is a factor of 1.5-2 larger than the one derived from astronomical observations, indicating a relative concentration of interstellar dust in the ISM close to the Sun compared to the $\sim 0.5 \mathrm{pc}$ LIC cloud length towards $\varepsilon$ CMa. The gas-to-dust mass ratio also varies by more than $30 \%$ over the nearest 3 pc. If ISM abundances are solar, the insitu and astronomical methods of determining $R_{\mathrm{g} / \mathrm{d}}$ are — generally —in better agreement, but interstellar absorption line data towards weakly reddened stars remain unexplained. These differences are not yet understood. The chemical composition of interstellar dust grains observed within the solar system thus provides a window on the chemical composition and homogeneity of the ISM.

The combination of absorption line data toward $\varepsilon \mathrm{CMa}$ and the modelled photoionization also lead to the conclusion that the LIC has a very interesting pattern of gas phase elemental abundances (Slavin and Frisch 2007, 2008): C appears to be substantially supersolar while $\mathrm{Fe}, \mathrm{Mg}$ and $\mathrm{Si}$ are subsolar. $\mathrm{O}$ and $\mathrm{N}$ are close to solar. This indicates that carbonaceous grains have been destroyed in the LIC while silicate grains have survived. The extra $\mathrm{C}$ in the gas has not been explained but may be evidence for a local enhancement of carbonaceous dust followed by grain destruction in a shock.

The masses of interstellar grains measured in-situ with the spacecraft detectors range from $10^{-18} \mathrm{~kg}$ to above $10^{-13} \mathrm{~kg}$. If we compare the mass distribution of these interstellar impactors with the dust mass distribution derived from astronomical observations, we find that the in-situ measurements overlap only with the largest masses observed astronomically. This is further supported by the radar measurements which revealed even bigger grains. These measurements imply that the intrinsic size distribution of interstellar grains in the LIC extends to sizes much larger than those grains which are detectable by astronomical observations (Frisch et al. 1999; Frisch and Slavin 2003; Landgraf et al. 2000; Grün and Landgraf 2000).

There are no direct observations of interstellar dust within $5 \mathrm{pc}$ and outside of the solar system. The observations of very weak starlight polarization towards nearby stars $(<40 \mathrm{pc})$ may originate from magnetically aligned dust grains close to the solar system. The observed polarization strength is consistent with the average interstellar density of $\sim 0.1 \mathrm{~cm}^{-3}$ over tens of parsecs in the upwind direction (Frisch 1990). The in-situ grains have a size distribution consistent with these classical dust grains.

Interstellar gas and dust couple through collisional processes, and through coupling of ions and charged grains to the interstellar magnetic field. Over distance scales of 100-500 pc, 
gas-dust coupling is demonstrated through the correlation of starlight-reddening dust grains (measured as color excess $\mathrm{E}(\mathrm{B}-\mathrm{V}))$ and interstellar hydrogen $\left(\mathrm{N}\left(\mathrm{H}^{0}\right)+2 \mathrm{~N}\left(\mathrm{H}_{2}\right)\right)($ Bohlin et al. 1978). For the multicloud structure observed within $5 \mathrm{pc}$, gas-dust coupling is not proven. The collisional lifetimes for classical dust grains (radii $\sim 0.2 \mu \mathrm{m}$ ) in the LIC are $\sim 0.3 \times 10^{6}$ years, during which time the LIC will move $\sim 5 \mathrm{pc}$ through local space. The gyroradius is $\sim 0.1 \mathrm{pc}$ in a field of $3 \mu \mathrm{G}$ (Grün and Landgraf 2000). The result will be magnetically captured dust grains that are collisionally destroyed over the lifetime of the cloud. Grün and Landgraf (2000) suggested that the small "classical" grains are replenished by the collisional destruction of larger dust grains. Alternatively, silicate grain destruction peaks near shock column densities of $\mathrm{N}(\mathrm{H}) \sim 6 \times 10^{17} \mathrm{~cm}^{-2}$ (Jones et al. 1994), allowing the breakdown of gas-dust coupling locally over $\sim 1$ pc length scales.

There are important consequences from the existence of the big particle population in the LIC. While particles observed by spacecraft couple to the interstellar medium on length scales of less than $1 \mathrm{pc}$ via electromagnetic interactions, more massive grains couple to the gas over much longer scales of 100 to 1000 pc (Grün and Landgraf 2000). Therefore, big interstellar meteor particles travel unaffected over much longer distances and may come directly from their source region.

\section{Outlook}

In 2007 and early 2008 Ulysses was in the inner solar system where interstellar grains cannot reliably be separated from interplanetary impactors but in mid-2008 the dust instrument could begin to monitor the interstellar grains again. Due to a serious failure in the power system on board, however, the spacecraft is not fully operational at the moment and the dust detector is switched off. It is presently unclear whether Ulysses can be put back into normal operation. Before the onboard failure occurred, a mission extension until 2011 was considered and would have provided interstellar dust data from the outer heliosphere again. With this extension the Ulysses measurements from the entire mission would have covered an almost entire 22-year solar cycle. But even now the Ulysses data is a unique set of dust measurements from interplanetary space for decades to come. Together with detailed modelling of the grain interaction with the IMF during the highly disordered solar maximum conditions we will hopefully be able to reveal the origin of the observed $30^{\circ}$ shift. If the shift turned out to be intrinsic, being potentially connected with a secondary population of interstellar grains, it would put strong constraints on the small-scale structure of the LIC. This would also be highly relevant for the interpretation of results from the Stardust mission which recently brought a sample of collected interstellar grains to Earth (A. Westphal 2006, priv. comm.), and for future dust astronomy space missions aiming at the in-situ analysis and sample return of interstellar dust (CosmicDUNE, SARIM; Grün et al. 2005; Srama et al. 2008).

Acknowledgements We thank the Ulysses project at ESA and NASA/JPL for effective and successful mission operations. This work has been supported by the Deutsches Zentrum für Luft- und Raumfahrt e.V. (DLR) under grants 50 0N 9107 and 50 QJ 9503. Support by Max-Planck-Institut für Kernphysik and MaxPlanck-Institut für Sonnensystemforschung is also gratefully acknowledged.

\section{References}

N. Altobelli, Monitoring of the interstellar dust stream in the inner solar system using data of different spacecraft. Ph.D. Thesis, Ruprecht-Karls-Universität Heidelberg (2004) 
N. Altobelli, S. Kempf, M. Landgraf, R. Srama, V. Dikarev, H. Krüger, G. Moragas-Klostermeyer, E. Grün, J. Geophys. Res. 108(A10), 7-1 (2003)

N. Altobelli, R. Moissl, H. Krüger, M. Landgraf, E. Grün, Planet. Space Sci. 52, 1287-1295 (2004)

N. Altobelli, S. Kempf, H. Krüger, M. Landgraf, R. Srama, E. Grün, in AIP Conf. Proc. 761: The Spectral Energy Distributions of Gas-Rich Galaxies: Confronting Models with Data (2005a), pp. 149-152

N. Altobelli, S. Kempf, H. Krüger, M. Landgraf, M. Roy, E. Grün, J. Geophys. Res. 110, 7102-7115 (2005b)

N. Altobelli, E. Grün, M. Landgraf, Astron. Astrophys. 448, 243-252 (2006)

W.J. Baggaley, J. Geophys. Res. 105(A5), 10,353-10,361 (2000)

W.J. Baggaley, L. Neslušan, Astron. Astrophys. 382, 1118-1124 (2002)

M. Baguhl, E. Grün, D.P. Hamilton, G. Linkert, R. Riemann, P. Staubach, Space Sci. Rev. 72, 471-476 (1995)

J.L. Bertaux, J.F. Blamont, Nature 262, 263-266 (1976)

R.C. Bohlin, B.D. Savage, J.F. Drake, Astrophys. J. 224, 132-142 (1978)

M.R. Collier, T.E. Moore, D. Simpson, A. Roberts, A. Szabo, S. Fuselier, P. Wurz, M.A. Lee, B.T. Tsurutani, Adv. Space Res. 34, 166-171 (2004)

A. Czechowski, I. Mann, J. Geophys. Res. 108(A10), 8038 (2003). doi:10.1029/2003JA009.917

B.T. Draine, Ann. Rev. Astron. Astrophys. 41, 241-289 (2003)

P.C. Frisch, in Physics of the Outer Heliosphere, ed. by S. Grzedzielski, D.E. Page, 1st COSPAR Colloquium, Warsaw (1990), pp. 19-22

P.C. Frisch, Space Sci. Rev. 86, 107 (1998)

P.C. Frisch, in The Galactic Environments of Nearby Cool Stars, ed. by L.M. Celnikier, J.T.T. Van (Editions Frontieres, 1999), pp. 3-10

P.C. Frisch, Astrophys. J. 593, 868-873 (2003)

P.C. Frisch, J.D. Slavin, Astrophys. J. 594, 844-858 (2003)

P.C. Frisch, J. Dorschner, J. Geiß, J.M. Greenberg, E. Grün, M. Landgraf, P. Hoppe, A.P. Jones, W. Krätschmer, T.J. Linde, G.E. Morfill, W.T. Reach, J. Slavin, J. Svestka, A. Witt, G.P. Zank, Astrophys. J. 525, 492-516 (1999)

E. Grün, M. Landgraf, J. Geophys. Res. 105(A5), 10,291-10,298 (2000)

E. Grün, H. Fechtig, J. Kissel, D. Linkert, D. Maas, J.A.M. McDonnell, G.E. Morfill, G.H. Schwehm, H.A. Zook, R.H. Giese, Astron. Astrophys. Suppl. 92, 411-423 (1992)

E. Grün, H.A. Zook, M. Baguhl, A. Balogh, S.J. Bame, H. Fechtig, R. Forsyth, M.S. Hanner, M. Horányi, J. Kissel, B.A. Lindblad, D. Linkert, G. Linkert, I. Mann, J.A.M. McDonnell, G.E. Morfill, J.L. Phillips, C. Polanskey, G.H. Schwehm, N. Siddique, P. Staubach, J. Svestka, A. Taylor, Nature 362, 428-430 (1993)

E. Grün, B.E. Gustafson, I. Mann, M. Baguhl, G.E. Morfill, P. Staubach, A. Taylor, H.A. Zook, Astron. Astrophys. 286, 915-924 (1994)

E. Grün, M. Baguhl, D.P. Hamilton, J. Kissel, D. Linkert, G. Linkert, R. Riemann, Planet. Space Sci. 43, 941-951 (1995). Paper I

E. Grün, P. Staubach, M. Baguhl, D.P. Hamilton, H.A. Zook, S.F. Dermott, B.A. Gustafson, H. Fechtig, J. Kissel, D. Linkert, G. Linkert, R. Srama, M.S. Hanner, C. Polanskey, M. Horányi, B.A. Lindblad, I. Mann, J.A.M. McDonnell, G.E. Morfill, G.H. Schwehm, Icarus 129, 270-288 (1997)

E. Grün, R. Srama, H. Krüger, S. Kempf, V. Dikarev, S. Helfert, G. Moragas-Klostermeyer, Icarus 174, 1-14 (2005)

C. Gry, E.B. Jenkins, Astron. Astrophys. 367, 617-628 (2001)

A.P. Jones, A.G.G.M. Tielens, D.J. Hollenbach, C.F. McKee, Astrophys. J. 433, 797-810 (1994)

H. Krüger, E. Grün, M. Landgraf, S.F. Dermott, H. Fechtig, B.A. Gustafson, D.P. Hamilton, M.S. Hanner, M. Horányi, J. Kissel, B. Lindblad, D. Linkert, G. Linkert, I. Mann, J.A.M. McDonnell, G.E. Morfill, C. Polanskey, G.H. Schwehm, R. Srama, H.A. Zook, Planet. Space Sci. 49, 1303-1324 (2001). Paper VII

H. Krüger, N. Altobelli, B. Anweiler, S.F. Dermott, V. Dikarev, A.L. Graps, E. Grün, B.A. Gustafson, D.P. Hamilton, M.S. Hanner, M. Horányi, J. Kissel, M. Landgraf, B. Lindblad, D. Linkert, G. Linkert, I. Mann, J.A.M. McDonnell, G.E. Morfill, C. Polanskey, G.H. Schwehm, R. Srama, H.A. Zook, Planet. Space Sci. 54, 932-956 (2006). Paper IX

H. Krüger, M. Landgraf, N. Altobelli, E. Grün, Space Sci. Rev. 130, 401-408 (2007)

M. Landgraf, Modellierung der Dynamik und Interpretation der In-situ-Messung interstellaren Staubs in der lokalen Umgebung des Sonnensystems. Ph.D. Thesis, Ruprecht-Karls-Universität Heidelberg (1998)

M. Landgraf, J. Geophys. Res. 105(A5), 10,303-10,316 (2000)

M. Landgraf, E. Grün, in Proceedings of the IAU Colloquium No. 166 on the Local Bubble and Beyond, ed. by D. Breitschwerdt, M.J. Freyberg, J. Trümper. Lecture Notes in Physics, vol. 506 (Springer, Heidelberg, 1998), pp. 381-384

M. Landgraf, K. Augustsson, E. Grün, B.A.S. Gustafson, Science 286, 2,319-2,322 (1999)

M. Landgraf, W.J. Baggaley, E. Grün, H. Krüger, G. Linker, J. Geophys. Res. 105(A5), 10,343-10,352 (2000) 
M. Landgraf, H. Krüger, N. Altobelli, E. Grün, J. Geophys. Res. 108, 5-1 (2003)

A. Li, J.M. Greenberg, Astron. Astrophys. 323, 566-584 (1997)

T.J. Linde, T.I. Gombosi, J. Geophys. Res. 105, 10,411-10,418 (2000)

I. Mann, H. Kimura, J. Geophys. Res. 105(A5), 10,317-10,328 (2000)

J.S. Mathis, J. Geophys. Res. 105, 10,269-10,278 (2000)

D.D. Meisel, D. Janches, J.D. Mathews, Astrophys. J. 567, 323-341 (2002)

G.E. Morfill, E. Grün, Planet. Space Sci. 27, 1283-1292 (1979)

H. Nakagawa, M. Bzowski, A. Yamazaki, H. Fukunishi, S. Watanabe, Y. Takahashi, M. Taguchi, in 36th COSPAR Scientific Assembly, vol. 36. COSPAR, Plenary Meeting (2006), pp. 1170-+

S. Redfield, J.L. Linsky, Astrophys. J. Suppl. 139, 439-465 (2002)

S. Redfield, J.L. Linsky, Astrophys. J. 602, 776-802 (2004a)

S. Redfield, J.L. Linsky, Astrophys. J. 613, 1004-1022 (2004b)

S. Redfield, J.L. Linsky, Astrophys. J. 673, 283-314 (2008)

J.D. Slavin, P.C. Frisch, Astrophys. J. Lett. 651, L37-L40 (2006)

J.D. Slavin, P. Frisch, Space Sci. Rev. 130, 409-414 (2007)

J.D. Slavin, P.C. Frisch, Astron. Astrophys. (2008, submitted). http://arxiv.org/abs/0704.0657

R. Srama, T. Stephan, E. Grün, N. Pailer, A. Kearsley, A.L. Graps, R. Laufer, P. Ehrenfreund, N. Altobelli, K. Altwegg, S. Auer, J. Baggaley, M. Burchell, J. Carpenter, L. Colangeli, F. Esposito, S. Green, H. Henkel, M. Horányi, A. Jäckel, S. Kempf, N. McBride, G. Moragas-Klostermeyer, H. Krüger, A. Srowig, M. Trieloff, P. Tsou, Z. Sternovsky, O. Zeile, H.-P. Röser, Exp. Astron. (2008, submitted)

A.D. Taylor, W.J. Baggaley, D.I. Steel, Nature 380, 323-325 (1996)

J. Tinbergen, Astron. Astrophys. 105, 53-64 (1982)

M. Witte, Astron. Astrophys. 426, 835-844 (2004)

M. Witte, H. Banaszkiewicz, H. Rosenbauer, Space Sci. Rev. 78(1/2), 289-296 (1996)

M. Witte, M. Banaszkiewicz, H. Rosenbauer, D. McMullin, Adv. Space Res. 34, 61-65 (2004)

H. Wolf, J. Rhee, O.E. Berg, in Interplanetary Dust and Zodiacal Light, ed. by H. Elsaesser, H. Fechtig. Lecture Notes in Physics, vol. 48 (Springer, Berlin, 1976), pp. 165-169

P. Wurz, M.R. Collier, T.E. Moore, D. Simpson, S. Fuselier, W. Lennartson, in Physics of the Outer Heliosphere, ed. by V. Florinski, N.V. Pogorelov, G.P. Zank. American Institute of Physics Conference Series, vol. 719 (2004), pp. 195-200 